Review Article

\title{
Radiation-Induced Noncancer Risks in Interventional Cardiology: Optimisation of Procedures and Staff and Patient Dose Reduction
}

\author{
Zhonghua Sun, ${ }^{1}$ Aini AbAziz, ${ }^{2}$ and Ahmad Khairuddin Md Yusof ${ }^{3}$ \\ ${ }^{1}$ Discipline of Medical Imaging, Department of Imaging and Applied Physics, Curtin University, P.O. Box U1987, Perth, \\ WA 6845, Australia \\ ${ }^{2}$ Department of Molecular Imaging and Nuclear Medicine, Universiti Kebangsaan Malaysia Medical Centre (UKMMC), \\ Jalan Yaakob Latif, Cheras, 56000 Kuala Lumpur, Malaysia \\ ${ }^{3}$ Department of Cardiology, National Heart Institute, 50300 Kuala Lumpur, Malaysia
}

Correspondence should be addressed to Aini AbAziz; ab_aziz_aini@hotmail.com

Received 30 April 2013; Revised 28 June 2013; Accepted 18 July 2013

Academic Editor: Eliseo Vano

Copyright (c) 2013 Zhonghua Sun et al. This is an open access article distributed under the Creative Commons Attribution License, which permits unrestricted use, distribution, and reproduction in any medium, provided the original work is properly cited.

\begin{abstract}
Concerns about ionizing radiation during interventional cardiology have been increased in recent years as a result of rapid growth in interventional procedure volumes and the high radiation doses associated with some procedures. Noncancer radiation risks to cardiologists and medical staff in terms of radiation-induced cataracts and skin injuries for patients appear clear potential consequences of interventional cardiology procedures, while radiation-induced potential risk of developing cardiovascular effects remains less clear. This paper provides an overview of the evidence-based reviews of concerns about noncancer risks of radiation exposure in interventional cardiology. Strategies commonly undertaken to reduce radiation doses to both medical staff and patients during interventional cardiology procedures are discussed; optimisation of interventional cardiology procedures is highlighted.
\end{abstract}

\section{Introduction}

Medical exposure from X-rays and nuclear medicine is the largest man-made source of radiation exposure, representing a mean effective dose of 1.0-3.0 mSv per head per year [1]. The worldwide population exposure from medical radiation has been shown to increase, and the use of procedures (both diagnostic and therapeutic) with a high radiation dose has been growing steadily [2-5]. Although interventional cardiac procedures account for $12 \%$ of all radiological examinations, they are responsible for delivering the highest radiation dose (up to $50 \%$ of the total collective effective dose) [6]. Thus, radiation exposure is a significant concern for interventional cardiologists and patients due to the increasing workloads and the complexity of procedures over the last decade $[7,8]$.

With fluoroscopy the patient is imaged in real time to guide minimally invasive procedures that form part of the diagnostic and interventional procedures, and this requires medical and technical staff to directly participate in the procedures. Patients undergoing interventional procedures in cardiology face radiation exposure in the order of a thousand or more times than that involved in conventional radiography [9]. Similarly, the interventional cardiologists encounter much more radiation than most other medical staff due to their working position being close to the X-ray beam and the patient (the source of scatter radiation). Therefore, interventional cardiologists must have a thorough knowledge of consequences of exposure to patients and personnel to ionizing radiation and methods of reducing staff and patient radiation exposure. Evaluation and followup of radiation doses received by the medical staff and patients should be considered an important part of quality assurance programmes for interventional cardiology procedures.

Radiation safety in the practice of interventional cardiology has been addressed by several professional bodies. In 2005, the American College of Cardiology (ACC) Foundation proposed the interventional cardiology guidelines which emphasized that physicians are responsible for minimizing 
the radiation injury hazard to their patients, professional staff, and themselves [10]. The UNSCEAR 2008 report states that fluoroscopic procedures represent the largest source of occupational exposure in medicine [11]. In 2009, the American Heart Association (AHA) Science Advisory recommended the reference doses of common cardiology examinations [12], and in 2010 the ACC committee also expressed the need for appropriate and optimal use of radiation techniques in cardiology [13].

This paper provides an overview of the radiation-induced noncancer risks during interventional cardiology procedures, with a focus on the radiation risks to interventional cardiologists and patients, as well as strategies commonly undertaken to reduce radiation exposure.

\section{Radiation-Induced Effects and Risks to Interventional Cardiologists and Other Medical Staff}

There are two main biological effects of ionizing radiation: stochastic effects, which include carcinogenic and genetic effects and deterministic effects (also called tissue reactions), which refer to an immediate and very predictable change to the tissue [14]. Stochastic effects are those for which the probability of an effect, rather than its severity, depends on the dose of radiation received [15]. Radiation-induced cancer and genetic effects are stochastic in nature and this has been well addressed in the literature [16]. Stochastic effects are believed not to have a dose threshold level because injury to a few cells, or even a single cell could theoretically result in the development of disease.

Deterministic effects occur when the dose exceeds a specific threshold. The severity of deterministic effects commonly increases with dose, as more cells are killed or damaged. Common examples of deterministic effects related to interventional cardiology are skin and hair changes [17], cataracts, and cardiovascular disease [18].

2.1. Radiation-Induced Cataracts. One of the most vital yet ill-defined effects associated with ionizing radiation exposure is the effect on the transparency of the eye lens, a pathology called radiation cataract. According to their anatomic location, cataract or presence of lens opacities can be classified into three main types: nuclear, cortical, and posterior subscapular [19]. Lens changes include small dots and vacuoles at early stage of cataract, and these lesions aggregate to form larger opacities at late stage of disease development. Although the sensitivity of the lens of the eye to high doses of ionizing radiation is well known, there exist uncertainties about the relation between radiation dose and cataracts. The National Council on Radiation Protection (NCRP) and the International Commission on Radiological Protection (ICRP) proposed guidelines on the view that cataractogenesis is a deterministic effect and requires a threshold radiation dose (currently 2 Gy) [14, 20]. However, radiation-induced cataracts are reported in populations exposed to much lower doses than the current standards, and this strongly suggests a stochastic hypothesis [21-23].
Occupational exposure to ionizing radiation and lens opacities has been reported for medical personnel, such as radiology technicians [24]. Earlier studies have demonstrated a significant increase in eye lens opacities among interventional cardiologists and medical staff in cardiac catheterization laboratories $[25,26]$. Later reports based on experiences from different countries indicated that risk of lens opacities among interventional cardiologists was at least twice that of unexposed groups [27, 28].

In April 2011, the ICRP revised its lifetime eye dose threshold for cataract induction downwards from $2000 \mathrm{mSv}$ to $500 \mathrm{mSv}$ and the occupational annual dose limit from $150 \mathrm{mSv}$ to $20 \mathrm{mSv}$ in a year, averaged over defined periods of 5 years, with no single year exceeding $50 \mathrm{mSv}[14,29]$. This recommendation is having an immediate impact on the new International Basic Safety Standard issued by the International Atomic Energy Agency (IAEA) and the upcoming Directive of the European Commission [30,31]. The impact of these new recommendations on the practice of international cardiology is considered significant, since interventional cardiologists are potentially at risk of developing radiationinduced cataracts, depending on their level of exposure and the number and complexity of invasive cardiac procedures $[32,33]$.

Some epidemiological studies have been published on the risk of cataracts in interventional cardiology. Junk et al. in their first report consisting of 59 volunteer participants (radiologists and cardiologists) observed that a high frequency of $37.3 \%$ participants having small paracentral dot-like opacities in the posterior subcapsular regions of the lens, consistent with early signs of radiation damage, and $8 \%$ had diagnosis of cataracts [34]. This has been confirmed by a recent report examining 54 cardiologists and 69 nurses and technicians, with lens change found in 50\% of interventional cardiologists and $41 \%$ of nurses and technicians compared with findings of similar lens changes in $<10 \%$ of controls [35].

The Occupational Cataracts and Lens Opacities in Interventional Cardiology (O'CLOC) study represents a large scale epidemiological study with the aim of testing the existence of an increased risk of radiation-induced cataracts among interventional cardiologists compared with a control group of cardiologists not exposed to X-rays $[33,36]$. Unlike these earlier studies, the O'CLOC study included 106 interventional cardiologists (including coronary interventional cardiologists and electrophysiologists) and 99 unexposed nonmedical workers. The study showed a significant excess risk of cataract for interventional cardiologists: $18 \%$ of posterior subcapsular lens opacities among interventional cardiologists were observed, while only $5 \%$ among control group were observed $(P<$ $0.05)$. Regarding cumulative eye lens dose, results were consistent with this excess risk. Overall, $29 \%$ of the interventional cardiologists and $20 \%$ of the electrophysiologists had a cumulative dose exceeding $500 \mathrm{mSv}$. These findings indicate that according to the revised ICRP lifetime eye dose threshold of $500 \mathrm{mSv},>25 \%$ of these cardiologists may already at risk of developing early radiation-induced cataracts. Furthermore, electrophysiologists may have had higher annual doses than cardiologists in recent years due to less use of eye protection equipment. These findings reinforce those reports based on 
small sample sizes and highlight the importance of increasing cardiologists' awareness of the regular use of radiation protection devices and the necessity of optimizing procedures for dose reduction.

The Optimization of RAdiation protection for MEDical staff (ORAMED) project is funded by EU-EURATOM within the $7^{\circ}$ Framework Programme with the aim of studying the dose received by operators in some selected practices of diagnostic and interventional radiology and nuclear medicine procedures $[37,38]$. The WP1 (working package) of the project is devoted to studying the eye lens and extremity doses in interventional radiology and cardiology. Early results of the ORAMED project showed that the highest eye lens doses were measured during embolization procedures [37]. With increasing workload and complexity of the interventional cardiology procedures, the annual eye lens doses would be estimated to be relatively higher or even exceed the dose limits.

In summary, there is evidence of radiation-induced cataract risk at lower doses than previously realised and following protracted exposure. Although studies provide additional evidence for radiation causing damage to the eye, even at low doses, most studies in the literature do not allow assessment of the clinical impact of the radiation associated opacities [29]. Ionizing radiation exposure has been identified to link to vision-impairing cataracts in the A-bomb survivors in a recent study [39], although further research is needed to focus on interventional cardiologists regarding radiation exposure and development of cataracts.

2.2. Radiation-Related Cardiovascular Diseases. Increased risk of cardiovascular diseases associated with ionizing radiation has received recent attention. Several studies have demonstrated the effects of ionizing radiation on hematologic parameters and immunologic function [40]; however, the question of whether radiation affects other physiologic phenomena, including arterial blood pressure, is still under debate despite continuous research efforts [41-43]. The key elements in radiation damage to vessels (microvessels in particular) are the endothelial cells [44]; however, much remains to be known about the intimate mechanisms of relationship between ionizing radiation and the endothelium damage. Experimental and human observations suggest that the endothelial cells are the most radioresponsive cells in the mesenchyma [45]. The initial response appears to be endothelial cell damage, leading to monocyte adhesion and transmigration into the subendothelial space. In the presence of elevated cholesterol levels, these invading monocytes transform into activated macrophages, which contribute to the formation of fatty streaks in the intima, resulting in the cascade of pathogenic changes that lead to radiation related heart disease [46-49]. Russo et al. analysed the response to chronic low-dose radiation by comparing 10 healthy interventional cardiologists with 10 unexposed controls by measuring hematological changes of redox state in lymphocytes [50]. The findings of their study demonstrate the association between low dose radiation and an altered redox balance which is manifested by an increase in hydrogen peroxide and adaptive cellular responses, although clinical meaning remains to be understood.

Early studies suggest that low-dose radiation can make human lymphocytes less susceptible to the genetic damage manifested as chromatic breakage induced by a subsequent high dose of X-rays [51,52]. Later mortality analysis of atomic bomb survivors shows that radiation exposure increases cardiovascular disease mortality, suggesting that ionizing radiation accelerates blood vessel degeneration [53]. Increased mortality risk was reported for heart disease, stroke, and respiratory diseases in the Life Span Study of atomic bomb survivors, with an excess relative risk for death from heart disease of 0.14 per sievert [54]. Dose above $0.5 \mathrm{~Gy}$ was found to be associated with an elevated risk of both stroke and heart disease [55].

Epidemiological data on low dose radiation-induced damage to cardiovascular system are scare and conflicting: an increased cardiovascular disease risk was reported from studies of early radiologists in USA [56] but not from radiologists in the UK [57]. Studies of radiologists and radiologic technologists in the USA [58], Canada [59], Japan [60], Denmark [61], and China [62] lacked individual doses. Hauptmann et al. reported excess mortality from cardiovascular system based on their data on the US radiologic technologists [58], while other studies have not provided detailed analyses of cardiovascular disease. Their results showed that, for deaths from ischemic heart disease based on an analysis of 633 radiologic technologists, the relative risks were $0.98,1.00$, and 1.22 during 1950-1959, 1940-1949, or before 1940, compared with 1960 or later.

Although mortality from cardiovascular diseases increased with radiation dose among atomic bomb survivors, other epidemiological data investigating the association between low doses of ionizing radiation and circulatory diseases have not provided clear evidence of such a relationship. Yamada et al. in their cross-sectional analysis of atomic bomb survivors found that aortic calcification increases with radiation dose, thus, suggesting linkage between low-dose radiation dose and atherosclerotic cardiovascular changes. Prevalence of mild aortic arch calcification was found in $26.2 \%$ for men and $31.9 \%$ for women. There is a significant correlation between aortic calcification and radiation dose with dose more than $0.5 \mathrm{~Gy}$ resulting in significantly higher percentage of severe calcification when compared to the dose value of less than $0.5 \mathrm{~Gy}$ [63]. This is consistent with the dose limits of $0.5 \mathrm{~Gy}$ provided by ICRP [14]. Thus, medical professionals should be aware that the absorbed dose threshold for cardiovascular disease might be as low as $0.5 \mathrm{~Gy}$ to the heart.

A longitudinal study of the relationship atomic bomb exposure and cardiovascular disease in the Adult Health Study (AHS) has shown dose related increases in the incidence of stroke and myocardial infarction and in the incidence or prevalence of hypertension, elevated cholesterol levels in the exposed subjects [64-67]. The findings of the epidemiological study of cardiovascular disease have been further confirmed by a recently published study conducted by Shimizu et al. with more than 50 years of followup of 86611 atomic bomb survivors [68]. The study provides the strongest 
evidence so far that radiation dose may increase the prevalence of stroke and heart disease at moderate dose levels (mainly 0.5-2 Gy), although robust confirmatory evidence from other studies is needed.

In summary, data on the association between chronic low dose radiation and cardiovascular diseases are currently limited. Epidemiological studies are needed to help clarify the possible mechanisms between radiation exposure and its effect on the microcardiovascular damage.

\section{Radiation-Induced Risks to Patients}

Interventional cardiology procedures such as coronary angiography, percutaneous transluminal coronary angioplasty (PTCA), radiofrequency ablation, electrophysiological study, and left ventriculography contribute a significant proportion of radiation dose to patients due to the long fluoroscopy times and high-quality images required. Radiation doses can vary substantially across the same cardiac angiographic and interventional procedures, which is often a result of varying complexities of examination or patient size but can be a consequence of technological or procedural preference.

Patient dosimetry methods and quantities currently used in interventional cardiology can be divided into three categories [69]: (1) dosimetry for stochastic risk evaluation, which is associated with the risk of cancer induction; (2) dosimetry for quality assurance, which addresses evaluation of the optimization level of interventional cardiology procedures in comparison with performance of equipment and operator skill or comparison of the practice among different clinical centres; (3) dosimetry for deterministic effects of radiation exposure, which is related to the risk of deterministic injuries occurring. Dose quantities such as DAP (dose area product), fluoroscopy time, cine time, and number of cine images are useful indicators for evaluation of optimization level of interventional procedures $[69,70]$. Effective dose is the most commonly used indicator in the assessment of diagnostic practice as it allows for estimation of the health risk due to stochastic effects of radiation.

3.1. Patient Dose. The radiation exposure to patients is determined by many factors, such as the X-ray equipment performance, the protocol used (e.g., frequency of cine frames, tube angulation, and the level of image quality), the operator skill and experience, the patient size, the interventional approach (e.g., femoral or radial technique), and several parameters related to the complexity and the nature of the intervention [71-74]. The Council Directive of the European Community 97/43 Euratom (MED) deals with the health protection of patients against risks of ionizing radiation associated with medical procedures and focuses on special procedures including interventional radiology [75]. Dose reference levels developed at the European level can be recommended in interventional cardiology examinations.

Delichas et al. found that the radiation dose to the patient is influenced by the individual characteristics of each interventional procedure [76]. The maximal annual dose values received by 9 cardiologists during 144 cardiac procedures in two hospitals in their study were found to be 1.9 and
$2.8 \mathrm{mSv}$, which are much higher than the reference levels defined by the European DIMOND approach [77]. Tsapaki et al. reported differences of radiation exposure to patients between cardiologists with various levels of experience. Their results showed that the mean DAP values for coronary angiography and PTCA were $34.3 \mathrm{~Gy} \times \mathrm{cm}^{2}$ and $55.3 \mathrm{~Gy} \times$ $\mathrm{cm}^{2}$ for cardiologists with more than 10 years of experience [78], while for cardiologists with less than 5 years of experience, the corresponding DAP values were $48.8 \mathrm{~Gy} \times \mathrm{cm}^{2}$ and $89.2 \mathrm{~Gy} \times \mathrm{cm}^{2}$, respectively. Bernardi et al. in their survey showed the correlation between radiation dose and types of interventional cardiology procedures with mean DAP being $65.8 \mathrm{~Gy} \times \mathrm{cm}^{2}, 93 \mathrm{~Gy} \times \mathrm{cm}^{2}$, and $116.7 \mathrm{~Gy} \times \mathrm{cm}^{2}$, corresponding to the simple, medium, and complex procedure groups [79]. These reports emphasize the importance of minimizing radiation dose to patients during interventional cardiology procedures by taking into account the operator's experience and complexity of the procedures.

In summary, patient radiation dose varies widely not only among different interventional cardiology procedures but also among published studies. Discrepancies of the available results in the literature are patient-, procedure-, cardiologist-, and fluoroscopic equipment-related. Interventional cardiology procedures can expose patients to high radiation doses, thus, efforts to minimize patient exposure should always be undertaken.

3.2. Radiation-Induced Skin Injury. Skin is the organ at greatest risk during complex interventional procedures. Skin changes such as erythema, ulcers, telangiectasia, and dermal atrophy are well-known deterministic effects of ionizing radiation [80-82]. Although commonly referred to as skin injuries, severe radiation injuries can extend into the subcutaneous fat and muscle [83]. Patients may face years of associated pain, multiple surgical procedures, and permanent disfigurement $[17,84]$. An early response (early transient ischemia) is noticed a few hours after doses of $>2 \mathrm{~Gy}$, when the exposed area is relatively large [14]. An actual skin dose in the 5-10 Gy range will always produce a noticeable injury with doses above 15 Gy leading to tissue being destroyed to a depth of a few centimetres and dermal necrosis $[14,85]$. To minimize this risk, evaluation and assessment of maximum skin dose in interventional cardiology procedures are of paramount importance and should be recommended in the daily practice, although it is very difficult to undertake [86, 87].

Case reports describing deterministic radiation injuries on patient skin are increasing in the literature, and the potential for deterministic effects in some instances may be of more concern than stochastic long-term risk. Padovani and colleagues reported that the frequency of skin injuries in patients undergoing interventional cardiac procedures was less than $0.03 \%$ [70]. Kato et al. in their recent study observed $1.5 \%$ of radiation skin injury in 400 consecutive interventional cardiac procedures [88]. The higher rate of radiation injury in Kato's study may be due to the inclusion of many complicated interventional procedures, which result in high radiation doses exceeding the safe threshold level for skin. Radiation 
injuries are often misdiagnosed due to the late occurrence of their signs and symptoms, usually weeks after interventional procedures. Major injuries continue to progress for many months after the procedure. In most cases skin injuries have been reported in relationship with not optimised or improper use of radiological equipment due to the lack of knowledge of interventional cardiologists of radiological image formation, radiology technology, and radiation protection rules. It is a legal requirement to report significant radiological incidents and accidents that during or as a direct result of using ionizing radiation for a medical procedure. However, in practice, such reporting system is hardly implemented in many countries.

The IAEA has set up its own international reporting system called SAFety in RADiological procedures (SAFRAD) which is anonymous, so that it can be used for dose monitoring and reporting [89]. The reporting system includes patients who are exposed to defined trigger levels or events in fluoroscopically guided diagnostic and interventional procedures in an international database. The Safety in Radiation Oncology (SAFRON) is in the process of being developed by the IAEA to compile reports of medical radiation "incidents" that put patients at risk [90], and the IAEA smart card project on SmartRad Track makes tracking patient exposure a reality [91].

Development of online methods, based on calculation of skin dose distribution on the patient's skin, could be useful to alert the cardiologists when the regional skin dose exceeds the threshold for deterministic injuries [92]. Balter and Moses introduced a dose managing program in interventional cardiology [85]. This program uses a significant dose (reference point dose of 5 Gy for coronary procedures) as an action trigger for additional documentation and followup, which is designed to set low enough dose value to minimize the probability of missing a clinical deterministic injury. Similarly, Faulkner et al. proposed trigger levels for different interventional cardiology procedures based on different field sizes (DAP trigger level ranges from 20 to $400 \mathrm{~Gy} \times \mathrm{cm}^{2}$ corresponding to field size from 10 to $200 \mathrm{~cm}^{2}$ ), which may be used to identify patients at the risk of deterministic injuries [93].

In summary, radiation injury to human skin occurs at actual skin doses as low as a few gray. The dose-response relationships for both early and late radiation-induced damage to the skin are significantly influenced by the exposure rate. Increasing the dose above the injury threshold increases the degree of injury and prolongs the healing process.

\section{Strategies to Reduce Radiation Risks to Interventional Cardiologists and Patients}

Radiation exposure of interventional cardiologists and patients is currently a major concern. The National Council on Radiation Protection and Measurements recommends intraprocedure announcements of air-kerma to occur at $1000 \mathrm{mGy}$ increments starting at $3000 \mathrm{mGy}$ and recommends that specific postprocedure management practices are implemented following procedures with considerable radiation dose levels $>5000 \mathrm{mGy}$ [94]. Cardiac patients are increasingly exposed to cumulative diagnostic and therapeutic techniques of cardiac imaging using ionizing radiation, such as coronary angiography (average effective dose 5-10 mSv), PTCA (7$20 \mathrm{mSv})$, and nuclear cardiology (6-15 mSv) [95].

Patient and staff dosimetry on paediatric interventional cardiology procedures is another issue as cardiologists generally need to stay closer to the patient in comparison with adult procedures [96]. A prominent feature in paediatric fluoroscopy and intervention is the large size of the image intensifiers relative to the size of the neonate, infant, or child. The image intensifier will completely cover the patient and therefore has the potential to increase radiation exposure if collimation is not used [97]. Reports involving the evaluation of paediatric cardiology procedures are limited. Ubeda et al. in their multicentre study indicated the large variability in paediatric interventional cardiology protocols and imaging parameters (including $\mathrm{kV}$ and $\mathrm{mA}$ ranges for fluoroscopy and cine modes), with measured scatter dose ranging from 0.8 to $12 \mathrm{mSvh}^{-1}$ at the eye position during fluoroscopy and cine modes if no protective tools are used [96].

Several aspects of radiation safety in the interventional cardiology have been proposed with effective dose reduction outcomes having been achieved. These include strategies of dose monitoring during the procedure, wearing protective devices, applying dose-reduction techniques, and implementing training and education programmes.

4.1. Dose Monitoring. It is necessary for interventional cardiologists to wear dosimeters on a regular basis. Occupational dosimetry is critical for the personal safety of interventional cardiologists. The ICRP and ACC recommend the use of two personal dosimeters, with one worn outside the apron at the shoulder or neck and the other worn under the apron at the waist $[6,98]$. In addition, special dosimeters could be used for special practices (e.g., a ring dosimeter for biliary drainage/stent procedure which involves delivery of high doses to the hands) to monitor doses to the skin, hands, feet, and the lens of eyes.

Martin conducted a review of the dose data from studies of radiology performed over the last 20 years which involved $\mathrm{X}$-ray procedures by radiology, cardiology, and other medical staff [99]. The doses in the studies reviewed vary by factors of 60-100 for similar procedures in different centres. As the number of interventional procedures is gradually increasing and the potential for medical staff to receive high doses, it is important to ensure that doses received by interventional cardiologists are monitored to check whether the protection devices are deployed effectively.

4.2. Use Protective Shielding and Wear Protective Devices. Equipment-mounted shielding includes protective drapes suspended from the table and from the ceiling. Ceiling-suspected shields can provide substantial dose reduction, especially to the unprotected areas such as head and neck. Undertable lead drapes reduce lower extremity dose substantially and should be used whenever possible [100]. They should always be employed, as they have been reported to significantly reduce operator dose [101].

It has been reported that interventional cardiologists received an average annual effective dose of $46.2 \mathrm{mSv}$ without 


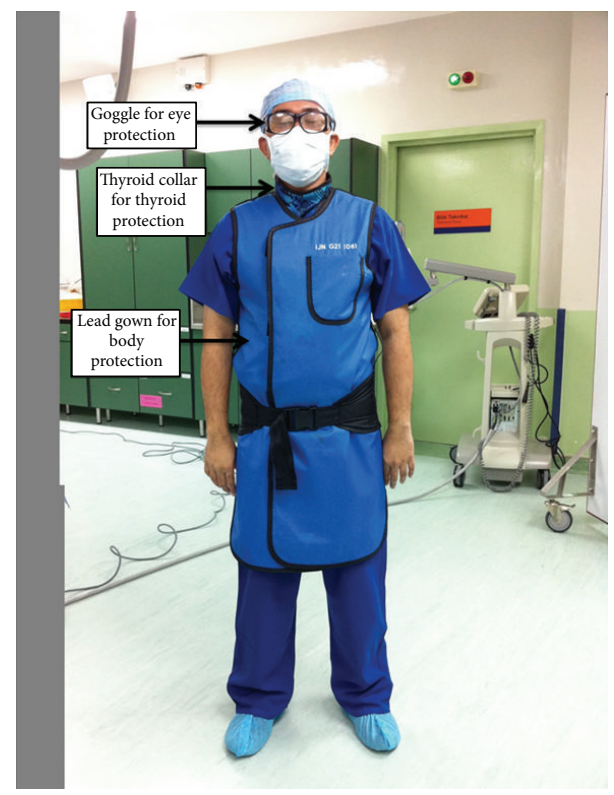

FIGURE 1: Wearing protective devices during interventional cardiology procedures.

wearing protective devices [102]. The dose can be reduced to $3.5 \mathrm{mSv}$ per year using a lead apron and to $1.7 \mathrm{mSv}$ per year using both a lead apron and a thyroid shield (Figure 1) [103]. Whereas the effective dose is routinely assessed by dosimetry, less attention is given to the local scatter radiation doses to unprotected parts of the body, especially the head and hands as cardiologists are also exposed to scatter radiation. Thus, wearing protective devices should be highly recommended during interventional cardiology procedures.

In cardiac intervention, radiation shields are widely used to reduce scatter radiation; however, the use of protective shields plays an essential role in optimizing protection during the interventional cardiology procedures (Figure 2). Fetterly et al. in their study reported that radiation shields must be thoughtfully placed and actively managed both before and during the procedure to be effective in providing substantial protection from radiation during interventional cardiology procedures [104]. Best practice guidelines for shield use are provided in their study with regard to upper body protection, lower body shields, or different approaches relating to different interventional procedures.

A leaded glass or plastic screen placed between the patient and the operator protects the operator's eyes, head, and neck. Leaded eye glasses with protective side shields provide more protection than eye glasses without these features [105]. Properly placed shields have been shown to reduce operator eye dose significantly $[106,107]$. These screens add no weight to the operator, eliminating the ergonomic consequences of the protective equipment; thus they can effectively replace both leaded eyewear [7]. Maeder et al. reported the effectiveness of reduction of scatter radiation to the eyes with use of a transparent lead glass screen, but minimal effects on the dose to the hands [106]. Therefore, additional efforts are required to reduce dose levels to both patients and operators.

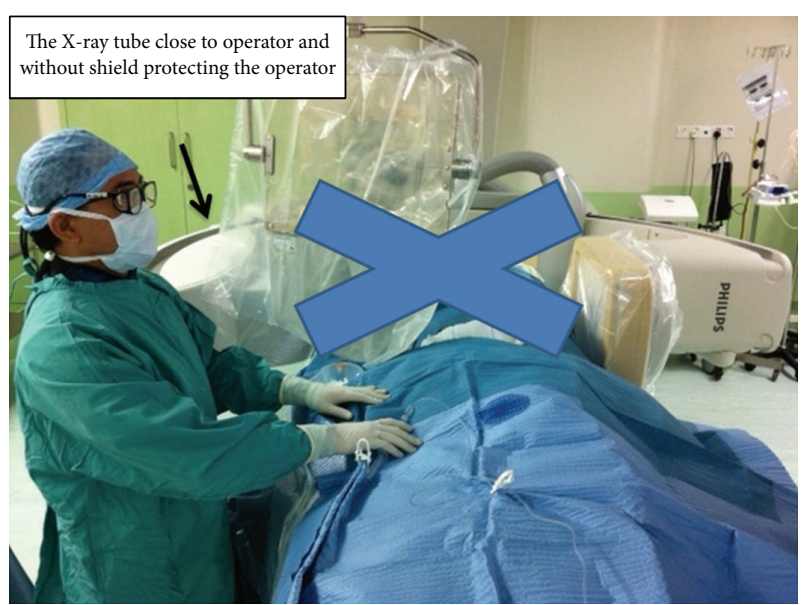

(a)

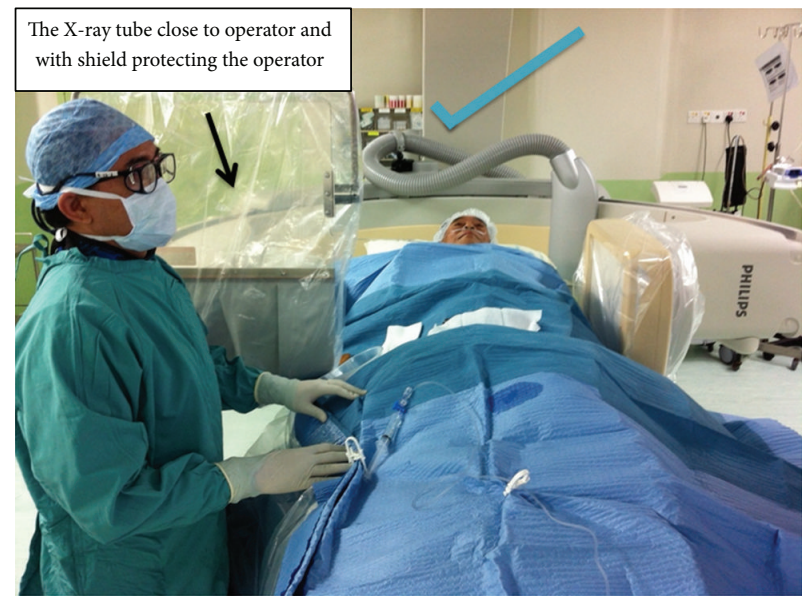

(b)

FIgURE 2: (a) The lateral projection is not recommended when the lead shield is not protecting the operator. (b) The lateral projection is recommended when the lead shield is protecting the operator.

4.3. Education and Training Programmes. It is important for the medical profession and other healthcare professionals to be aware of the hazards from radiation in order to avoid the unnecessary risks to the population as a whole. Lack of knowledge may result in more ionizing radiation imaging examinations being requested when other nonradiation tests could be performed or when different lower-dose imaging tests could be performed. This is particularly important for interventional cardiology procedures as they deliver high doses to medical staff and patients. As such, the need for education and training in radiological protection is more compelling.

The European Commission and ICRP have addressed the importance of training in radiological protection, publishing guidelines with specific recommendations for accreditation of training programmes for interventional procedures [108, 109]. ICRP Publication 113 recommends that training in radiological protection is included in the quality assurance programme, with special attention to training given to fellows and residents [109]. The guideline provided by European Commission suggests specific learning objectives and 20-30 hours of training for interventional cardiologists. Much effort 
TABLE 1: Dose reduction techniques that are commonly used in interventional cardiology procedures.

\begin{tabular}{ll}
\hline Techniques used in interventional cardiology & Corresponding functions \\
\hline Minimize use of fluoroscopy time and use low fluoroscopy mode & Reduce staff and patient dose \\
Number of fluorographic images & Reduce staff and patient dose \\
Image-chain geometry & Reduce patient dose \\
Collimation of the radiation field & Decrease the level of scatter dose \\
Medical staff position in a low-scatter area & Reduce staff dose \\
Wear protective shielding & Reduce radiation dose to eye lens and other organs \\
Fluoroscopic imaging equipment comply with International & Dose-reduction technology is incorporated into the imaging systems \\
Electrotechnical Commission [119] & Increase awareness of radiation protection and dose reduction \\
Obtain appropriate training provided by professional bodies & Know and monitor your own dose \\
Wear personal dosimeter & Monitor clinical practice and radiation dose \\
\hline
\end{tabular}

has been made over the last decade to produce training materials to help improvement of radiation protection in interventional cardiology procedures, with successful outcomes having been achieved $[85,95,110]$. The educational programme has been shown to be effective at improving compliance with the radiation badge monitoring programme [111]. Cardiology scientific societies should promote training activities in radiation protection to maintain a high level of radiation safety in the practice of interventional cardiology. The Guidance Document developed as work package 3 of the MEDRAPET project is expected to be used as the basis for curricula of radiation protection courses [112].

There is an urgent need to implement and propagate widely the training programs in interventional cardiology such as the IAEA initiated radiation cataract study called Retrospective Evaluation of Lens Injuries and Dose (RELID), which is organized in collaboration with professional societies of cardiologists in many countries [113].

4.4. Dose Reduction Techniques. Operational measures play an important role in improving radiation protection and dose reduction to both medical staff and patients. Decreasing patient dose will result in a proportional decrease in scatter dose to the operator; therefore, techniques that reduce patient dose will generally reduce occupational dose. A practical advice to reduce or minimize the occupational radiation dose has been recently proposed by the Cardiovascular and Interventional Society of Europe (CIRSE) [101]. Table 1 summarises various dose-reduction techniques and the corresponding functions.

One of the most important radiation protection measures is to increase patient's distance from the radiation source. Working at $80 \mathrm{~cm}$ from the isocenter instead of $40 \mathrm{~cm}$ can decrease scattered dose to approximately $25 \%$ of the original dose [114]. Variable dose values associated with radial versus femoral artery access have been reported in the literature $[115,116]$. Fetterly et al. demonstrated that patient dose was reduced simultaneously with increased utilization of radial access [104]. Other technical alterations provide the potential for systematic dose reduction, including a change from continuous fluoroscopy to pulsed fluoroscopy modes; reduction in fluoroscopy/acquisition frame rate from 30 frames/s to $7.5 / 15$ frames/s; collimation of the radiation field to decrease the level of scattered dose; improved X-ray image detection and display systems; and increased use of metallic X-ray beam spectral filters for both fluoroscopy and acquisition imaging $[104,114]$. Improved image processing within the fluoroscopic unit can compensate to a greater extent for the reduced image quality due to decreased exposure levels.

Diagnostic reference levels (DRLs) are radiation dose values for specific examinations that should not be consistently exceeded when good practice is in place and if regularly exceeded remedial action should be sought. DRLs are used to help avoid radiation dose to the patient that does not contribute to the medical imaging task. They are intended to provide guidance on what is achievable with current good practice rather than optimum performance and help to identify unusually high radiation doses or exposure levels [7]. These are legal requirements in a number of jurisdictions $[117,118]$ and have been shown to be very effective in reducing dose and dose variations for a variety of investigations since their introduction two decades ago.

\section{Summary and Conclusion}

In recent years, intensive efforts have been initiated to reduce the radiation dose associated with interventional cardiology. It has been become a routine practice for publications addressing cardiac intervention to report radiation doses. There is increasing concern about the potential deleterious effects from radiation arising from intervention cardiology due to two reasons: first, cardiac procedure volumes have grown tremendously. Second, the radiation doses received by interventional cardiologists and patients can vary by more than an order of magnitude for the same type of procedure. Increased workload, complexity of the interventional procedures, and acute patient conditions contribute significantly to the amount of radiation exposure to both patients and medical staff.

Noncancer risks of radiation in interventional cardiology that have been discussed in different scenarios emphasize the importance of reducing radiation dose to patients 
and medical staff. This can be achieved through implementing necessary strategies such as continual improvements in protocols and equipment, implementation of guidelines proposed by professional bodies into daily practice and attending training programmes to ensure best practice. Epidemiological studies involving a large cohort of individuals exposed to ionizing radiation will provide us with a full picture as to the true effects of radiation exposure from interventional cardiology. A final general recommendation is that being aware of the radiological protection of your patient will also be improving your own occupational protection.

\section{References}

[1] F. A. Mettler Jr., M. Bhargavan, K. Faulkner et al., "Radiologic and nuclear medicine studies in the United States and worldwide: frequency, radiation dose, and comparison with other radiation sources, 1950-2007," Radiology, vol. 253, no. 2, pp. 520-531, 2009.

[2] K. P. Kim, D. L. Miller, S. Balter et al., "Occupational radiation doses to operators performing cardiac catheterization procedures," Health Physics, vol. 94, no. 3, pp. 211-227, 2008.

[3] J. Vassileva, E. Vano, C. Ubeda, M. Rehani, and R. Zotova, "Impact of the X-ray system settings on patient dose and image quality; a case study with two interventional cardiology systems," Radiation Protection Dosimetry, 2013.

[4] E. Vano, R. Sanchez, J. M. Fernandez et al., "Patient dose reference levels for interventional radiology: a national approach," CardioVascular and Interventional Radiology, vol. 32, no. 1, pp. 19-24, 2009.

[5] A. J. Einstein, K. W. Moser, R. C. Thompson, M. D. Cerqueira, and M. J. Henzlova, "Radiation dose to patients from cardiac diagnostic imaging," Circulation, vol. 116, no. 11, pp. 1290-1305, 2007.

[6] E. Picano, E. Vano, R. Semelka, and D. Regulla, "The American College of Radiology white paper on radiation dose in medicine: deep impact on the practice of cardiovascular imaging," Cardiovascular Ultrasound, vol. 5, article 37, 2007.

[7] C. Cousins, D. L. Miller, G. Bernardi et al., "ICRP publication 120: radiological protection in cardiology," Annals of ICRP, vol. 42, pp. 1-125, 2013.

[8] E. Vano and K. Faulkner, "ICRP special radiation protection issues in interventional radiology, digital and cardiac imaging," Radiation Protection Dosimetry, vol. 117, no. 1-3, pp. 13-17, 2006.

[9] M. M. Rehani and P. Ortiz-Lopez, "Radiation effects in fluoroscopically guided cardiac interventions-keeping them under control," International Journal of Cardiology, vol. 109, no. 2, pp. 147-151, 2006.

[10] J. W. Hirshfeld Jr., S. Baiter, J. A. Brinker et al., "ACCF/AHA/ HRS/SCAI clinical competence statement on physician knowledge to optimize patient safety and image quality in fluoroscopically guided invasive cardiovascular procedures: a report of the American College of Cardiology Foundation/American Heart Association/American College of Physicians Task Force on Clinical Competence and Training," Circulation, vol. 111, no. 4, pp. 511-532, 2005.

[11] United Nations Scientific Committee on the Effects of Atomic Radiation. Sources and effects of ionizing radiation, "UNSCEAR 2008 report to the General Assembly," vol 1. Annex A. Medical Radiation Exposures, United Nations, New York, NY, USA, 2008, http://www.unscear.org/.
[12] T. C. Gerber, J. Jeffrey Carr, A. E. Arai et al., "Ionizing radiation in cardiac imaging: a science advisory from the American Heart Association Committee on cardiac imaging of the council on clinical cardiology and committee on cardiovascular imaging and intervention of the council on cardiovascular radiology and intervention," Circulation, vol. 119, no. 7, pp. 1056-1065, 2009.

[13] R. Brindis and P. S. Douglas, "President's page: the ACC encourages multi-pronged approach to radiation safety," Journal of the American College of Cardiology, vol. 56, no. 6, pp. 522-524, 2010.

[14] The International Commission on Radiological Protection, "Publication 118: ICRP Statement on tissue reactions and early and late effects of radiation in normal tissues and organs: threshold doses for tissue reactions and other non-cancer effects of radiation in a radiation protection context," Annals of ICRP, vol. 41, pp. 1-322, 2012.

[15] The International Commission on Radiological Protection, "The 2007 recommendations of the International Commission on Radiological Protection. ICRP publication 103," Annals of ICRP, vol. 37, pp. 1-332, 2007.

[16] Committee to Assess Health Risks from Exposure to Low Levels of Ionizing Radiation, Health Risks from Exposure to Low Levels of Ionizing Radiation: BEIR VII Phase 2, The National Academies Press, Washington, DC, USA, 2006.

[17] S. Balter, J. W. Hopewell, D. L. Miller, L. K. Wagner, and M. J. Zelefsky, "Fluoroscopically guided interventional procedures: a review of radiation effects on patients' skin and hair," Radiology, vol. 254, no. 2, pp. 326-341, 2010.

[18] A. J. Einstein, "Effects of radiation exposure from cardiac imaging: how good are the data?" Journal of the American College of Cardiology, vol. 59, no. 6, pp. 553-565, 2012.

[19] S. Jacob, S. Boveda, O. Bar et al., "Interventional cardiologists and risk of radiation-induced cataract: results of a French multicentre observational study," International Journal of Cardiology, 2012.

[20] National Council on Radiation Protection and Measurement, "Limitation of exposure to ionizing radiation," NCRP Report 116, Bethesda, Md, USA, 1993.

[21] N. Kleiman, "Radiation cataract," in Radiation Protection: New Insights in Radiation Risk and Basic Safety Standards, European Commission proceedings of the Working Party on Research Implications on Health and Safety Standards of the Article 31 group of Experts, RP-145, pp. 81-95, European Commission. Directorate General for Energy and Transport, Luxembourg, Belgium, 2007, http://ec.europa.eu/energy/nuclear/radiation_ protection/doc/publication/145.pdf.

[22] B. V. Worgul, Y. I. Kundiev, N. M. Sergienko et al., "Dose related incidence of radiation cataract in a six year follow-up of a cohort of the Chernobyl clean-up workers," Investigative Ophthalmology and Visual Science, vol. 44, supplement 2, p. 4241, 2003.

[23] B. V. Worgul, Y. I. Kundiyev, N. M. Sergiyenko et al., "Cataracts among Chernobyl clean-up workers: implications regarding permissible eye exposures," Radiation Research, vol. 167, no. 2, pp. 233-243, 2007.

[24] G. Chodick, N. Bekiroglu, M. Hauptmann et al., "Risk of cataract after exposure to low doses of ionizing radiation: a 20year prospective cohort study among US radiologic technologists," American Journal of Epidemiology, vol. 168, no. 6, pp. 620631, 2008.

[25] E. Vano, N. J. Kleiman, A. Duran, M. M. Rehani, D. Echeverri, and M. Cabrera, "Radiation cataract risk in interventional 
cardiology personnel," Radiation Research, vol. 174, no. 4, pp. 490-495, 2010.

[26] O. Ciraj-Bjelac, M. M. Rehani, K. H. Sim, H. B. Liew, E. Vano, and N. J. Kleiman, "Risk for radiation-induced cataract for staff in interventional cardiology: is there reason for concern?" Catheterization and Cardiovascular Interventions, vol. 76, no. 6, pp. 826-834, 2010.

[27] S. Mrena, T. Kivelä, P. Kurttio, and A. Auvinen, "Lens opacities among physicians occupationally exposed to ionizing radiation-a pilot study in Finland," Scandinavian Journal of Work, Environment and Health, vol. 37, no. 3, pp. 237-243, 2011.

[28] O. Ciraj-Bjelac, M. Rehani, A. Minamoto A et al., "Radiationinduced eye lens changes and risk for cataract in interventional cardiology," Cardiology, vol. 123, pp. 168-171, 2012.

[29] S. Bouffler, E. Ainsbury, P. Gilvin, and J. Harrison, "Radiationinduced cataracts: the Health Protection Agency's response to the ICRP statement on tissue reactions and recommendations on the dose limit for the eye lens," Journal of Radiation Protection, vol. 32, pp. 479-488, 2012.

[30] International Atomic Energy Agency, "Radiation Protection and Safety of Radiation Sources: International Basic Safety Standards (BSS) - Interim Edition, IAEA Safety Standards Series GSR Part3 (Interim)," IAEA, Vienna, Austria, 2011.

[31] European Commission, "Proposal for a Council Directive laying down basic safety standards for protection against the dangers arising from exposure to ionising radiation," 2012, http://ec.europa.eu/energy/nuclear/radiation_protection/doc/ 2012_com_242.pdf.

[32] M. M. Rehani, E. Vano, O. Ciraj-Bjelac, and N. J. Kleiman, "Radiation and cataract," Radiation Protection Dosimetry, vol. 147, no. 1-2, pp. 300-304, 2011.

[33] S. Jacob, L. Donadille, C. Maccia et al., "Eye lens radiation exposure to interventional cardiologists: a retrospective assessment of cumulative doses," Radiation Protection Dosimetry, vol. 153, pp. 282-293, 2013.

[34] A. Junk, Z. Haskal, and B. Worgul, "Cataract in interventional radiology-an occupational hazard?" Investigative Ophthalmology \& Visual Science, vol. 45, E-Abstract 388, 2004.

[35] E. Vano, N. J. Kleiman, A. Duran et al., "Radiation-associated lens opacities in catheterization personnel: results of a survey and direct assessments," Journal of Vascular and Interventional Radiology, vol. 24, pp. 197-204, 2013.

[36] S. Jacob, M. Michel, C. Spaulding et al., "Occupational cataracts and lens opacities in interventional cardiology (O'CLOC study): are X-Rays involved? Radiation-induced cataracts and lens opacities," BMC Public Health, vol. 10, article 537, 2010.

[37] J. Domienik, M. Brodecki, E. Carinou et al., "Extremity and eye lens doses in interventional radiology and cardiology procedures: first results of the oramed project," Radiation Protection Dosimetry, vol. 144, no. 1-4, Article ID ncq508, pp. 442-447, 2011.

[38] G. Gualdrini, F. Mariotti, S. Wach et al., "Eye lens dosimetry: task 2 within the oramed project," Radiation Protection Dosimetry, vol. 144, no. 1-4, Article ID ncr011, pp. 473-477, 2011.

[39] K. Neriishi, E. Nakashima, M. Akahoshi et al., "Radiation dose and cataract surgery incidence in atomic bomb survivors, 19862005," Radiology, vol. 265, pp. 167-174, 2012.

[40] S. C. Finch and C. A. Finch, "Summary of the studies at ABCCRERF concerning the late hematological effects of Atomic Bomb exposure in Hiroshima and Nagasaki," Tech. Rep. 23-88, Radiation Effects Research Foundation, 1988.
[41] A. Tsuya, Y. Wakano, and M. Otake, "Capillary microscopic observation on the superficial minute vessels of atomic bomb survivors, 1956-1957," Radiation Research, vol. 46, no. 1, pp. 199216, 1971.

[42] S. Switzer, "ABCC-JNIH Adult Health Study Hiroshima, 195859. Hypertension and ischemic heart disease," Radiation Effects Research Foundation, Hiroshima, Japan, 1961.

[43] K. Yano and S. Ueda, "Cardiovascular disease in relation to exposure to ionizing radiation," Radiation Effects Research Foundation, Hiroshima, Japan, 1962.

[44] L. F. Fajardo, "The unique physiology of endothelial cells and its implications in radiobiology," Frontiers of Radiation Therapy and Oncology, vol. 23, pp. 96-112, 1989.

[45] L. F. Fajardo, "Is the pathology of radiation injury different in small versus large blood vessels?" Cardiovascular Radiation Medicine, vol. 1, no. 1, pp. 108-110, 1999.

[46] J. Vos, M. W. Aarnoudse, F. Dijk, and H. B. Lamberts, "On the cellular origin and development of atheromatous plaques. A light and electron microscopic study of combined X-ray and hypercholesterolemia-induced atheromatosis in the carotid artery of the rabbit," Virchows Archiv B, vol. 43, no. 1, pp. 1-16, 1983.

[47] J. H. Hendry, M. Akahoshi, L. S. Wang, S. E. Lipshultz, F. A. Stewart, and K. R. Trott, "Radiation-induced cardiovascular injury," Radiation and Environmental Biophysics, vol. 47, no. 2, pp. 189-193, 2008.

[48] S. Schultz-Hector and K.-R. Trott, "Radiation-induced cardiovascular diseases: is the epidemiologic evidence compatible with the radiobiologic data?" International Journal of Radiation Oncology Biology Physics, vol. 67, no. 1, pp. 10-18, 2007.

[49] S. R. Basavaraju and C. E. Easterly, "Pathophysiological effects of radiation on atherosclerosis development and progression, and the incidence of cardiovascular complications," Medical Physics, vol. 29, no. 10, pp. 2391-2403, 2002.

[50] G. L. Russo, I. Tedesco, M. Russo, A. Cioppa, M. G. Andreassi, and E. Picano, "Cellular adaptive response to chronic radiation exposure in interventional cardiologists," European Heart Journal, vol. 33, no. 3, pp. 408-414, 2012.

[51] J. D. Shadley and S. Wolff, "Very low doses of X-rays can cause human lymphocytes to become less susceptible to ionizing radiation," Mutagenesis, vol. 2, no. 2, pp. 95-96, 1987.

[52] L. M. Stoilov, L. H. F. Mullenders, F. Darroudi, and A. T. Natarajan, "Adaptive response to DNA and chromosomal damage induced by X-rays in human blood lymphocytes," Mutagenesis, vol. 22, no. 2, pp. 117-122, 2007.

[53] Y. Shimizu, H. Kato, W. J. Schull, and D. G. Hoel, "Studies of the mortality of A-bomb survivors-9. Mortality, 1950-1985: part 3. Noncancer mortality based on the revised doses (DS86)," Radiation Research, vol. 130, no. 2, pp. 249-266, 1992.

[54] D. L. Preston, Y. Shimizu, D. A. Pierce, A. Suyama, and K. Mabuchi, "Studies of mortality of atomic bomb survivorsreport 13: solid cancer and noncancer disease mortality: 19501997," Radiation Research, vol. 160, no. 4, pp. 381-407, 2003.

[55] K. Ozasa, Y. Shimizu, R. Sakata et al., "Risk of cancer and noncancer diseases in the atomic bomb survivors," Radiation Protection Dosimetry, vol. 146, no. 1-3, pp. 272-275, 2011.

[56] M. M. Doody, J. S. Mandel, J. H. Lubin, and J. D. Boice Jr., "Mortality among United States radiologic technologists, 19261990," Cancer Causes and Control, vol. 9, no. 1, pp. 67-75, 1998.

[57] A. Berrington, S. C. Darby, H. A. Weiss, and R. Doll, "100 years of observation on British radiologists: mortality from cancer 
and other causes 1897-1997," British Journal of Radiology, vol. 74, no. 882, pp. 507-519, 2001.

[58] M. Hauptmann, A. K. Mohan, M. M. Doody, M. S. Linet, and K. Mabuchi, "Mortality from diseases of the circulatory system in radiologic technologists in the United States," American Journal of Epidemiology, vol. 157, no. 3, pp. 239-248, 2003.

[59] J. P. Ashmore, D. Krewski, J. M. Zielinski, H. Jiang, R. Semenciw, and P. R. Band, "First analysis of mortality and occupational radiation exposure based on the National Dose Registry of Canada," American Journal of Epidemiology, vol. 148, no. 6, pp. 564-574, 1998.

[60] S. Yoshinaga, T. Aoyama, Y. Yoshimoto, and T. Sugahara, "Cancer mortality among radiological technologists in Japan: updated analysis of follow-up data from 1969 to 1993," Journal of Epidemiology, vol. 9, no. 2, pp. 61-72, 1999.

[61] M. Andersson, G. Engholm, K. Ennow, K. A. Jessen, and H. H. Storm, "Cancer risk among staff at two radiotherapy departments in Denmark," British Journal of Radiology, vol. 64, no. 761, pp. 455-460, 1991.

[62] J.-X. Wang, J. D. Boice Jr., B.-X. Li, L.-Y. Zhang, and J. F. Fraumeni Jr., "Cancer among medical diagnostic X-ray workers in China," Journal of the National Cancer Institute, vol. 80, no. 5, pp. 344-350, 1988.

[63] M. Yamada, F. L. Wong, S. Fujiwara, M. Akahoshi, and G. Suzuki, "Noncancer disease incidence in atomic bomb survivors, 1958-1998," Radiation Research, vol. 161, no. 6, pp. 622$632,2004$.

[64] H. Sasaki, F. L. Wong, M. Yamada, and K. Kodama, "The effects of aging and radiation exposure on blood pressure levels of atomic bomb survivors," Journal of Clinical Epidemiology, vol. 55, no. 10, pp. 974-981, 2002.

[65] F. Kasagi, K. Kodama, M. Yamada et al., "An association between the prevalence of isolated hypertension and radiation dose in the Adult Health Study," Nagasaki Medical Journal, vol. 67, pp. 479-482, 1992.

[66] F. L. Wong, M. Yamada, H. Sasaki, K. Kodama, and Y. Hosoda, "Effects of radiation on the longitudinal trends of total serum cholesterol levels in the atomic bomb survivors," Radiation Research, vol. 151, no. 6, pp. 736-746, 1999.

[67] M. Yamada, K. Naito, F. Kasagi, N. Masunari, and G. Suzuki, "Prevalence of atherosclerosis in relation to atomic bomb radiation exposure: an RERF Adult Health Study," International Journal of Radiation Biology, vol. 81, no. 11, pp. 821-826, 2005.

[68] Y. Shimizu, K. Kodama, N. Nishi et al., "Radiation exposure and circulatory disease risk: Hiroshima and Nagasaki atomic bomb survivor data, 1950-2003," British Medical Journal, vol. 340, no. 7739, article 193, 2010.

[69] I. Pantos, G. Patatoukas, D. G. Katritsis, and E. Efstathopoulos, "Patient radiation doses in interventional cardiology procedures," Current Cardiology Reviews, vol. 5, no. 1, pp. 1-11, 2009.

[70] R. Padovani, G. Bernardi, E. Quia et al., "Retrospective evaluation of occurrence of skin injuries in interventional cardiac procedures," Radiation Protection Dosimetry, vol. 117, no. 1-3, pp. 247-250, 2006.

[71] E. Kuon, C. Glaser, and J. B. Dahm, "Effective techniques for reduction of radiation dosage to patients undergoing invasive cardiac procedures," British Journal of Radiology, vol. 76, no. 906, pp. 406-413, 2003.

[72] A. L. Clark, A. G. Brennan, L. J. Robertson, and J. D. Mcarthur, "Factors affecting patient radiation exposure during routine coronary angiography in a tertiary referral centre," British Journal of Radiology, vol. 73, no. 866, pp. 184-189, 2000.
[73] F. Larrazet, A. Dibie, F. Philippe, R. Palau, R. Klausz, and F. Laborde, "Factors influencing fluoroscopy time and dosearea product values during and hoc one-vessel percutaneous coronary angioplasty," British Journal of Radiology, vol. 76, no. 907, pp. 473-477, 2003.

[74] R. Padovani, G. Bernardi, M. R. Malisan, E. Vañó, G. Morocutti, and P. M. Fioretti, "Patient dose related to the complexity of interventional cardiology procedures," Radiation Protection Dosimetry, vol. 94, no. 1-2, pp. 189-192, 2001.

[75] D. Teunen, "The European Directive on health protection of individuals against the dangers of ionising radiation in relation to medical exposures (97/43/EURATOM)," Journal of Radiological Protection, vol. 18, no. 2, pp. 133-137, 1998.

[76] M. G. Delichas, K. Psarrakos, K. Hatziioannou et al., “The dependence of patient dose on factors relating to the technique and complexity of Interventional Cardiology procedures," Physica Medica, vol. 21, no. 4, pp. 153-157, 2005.

[77] V. Neofotistou, E. Vano, R. Padovani et al., "Preliminary reference levels in interventional cardiology," European Radiology, vol. 13, no. 10, pp. 2259-2263, 2003.

[78] V. Tsapaki, S. Kottou, E. Vano et al., "Patient dose values in a dedicated Greek cardiac centre," British Journal of Radiology, vol. 76, no. 910, pp. 726-730, 2003.

[79] G. Bernardi, R. Padovani, G. Morocutti et al., "Clinical and technical determinants of the complexity of percutaneous transluminal coronary angioplasty procedures: analysis in relation to radiation exposure parameters," Catheterization and Cardiovascular Interventions, vol. 51, pp. 1-9, 2000.

[80] T. R. Koenig, D. Wolff, F. A. Mettler, and L. K. Wagner, "Skin injuries from fluoroscopically guided procedures-part 1, characteristics of radiation injury," American Journal of Roentgenology, vol. 177, no. 1, pp. 3-11, 2001.

[81] T. R. Koenig, F. A. Mettler, and L. K. Wagner, "Skin injuries from fluoroscopically guided procedures-part 2, review of 73 cases and recommendations for minimizing dose delivered to patient," American Journal of Roentgenology, vol. 177, no. 1, pp. 13-20, 2001.

[82] Centers for Disease Control and Prevention, "Cutaneous radiation injury: fact sheet for physicians," Centers for Disease Control and Prevention, 2006, http://www.bt.cdc.gov/radiation/ arsphysicianfactsheet.asp.

[83] J. L. Monaco, K. Bowen, P. N. Tadros, and P. D. Witt, "Iatrogenic deep musculocutaneous radiation injury following percutaneous coronary intervention," Journal of Invasive Cardiology, vol. 15, no. 8, pp. 451-453, 2003.

[84] T. H. Frazier, J. B. Richardson, V. C. Fabré, and J. P. Callen, "Fluoroscopy-induced chronic radiation skin injury: a disease perhaps often overlooked," Archives of Dermatology, vol. 143, no. 5, pp. 637-640, 2007.

[85] S. Balter and J. Moses, "Managing patient dose in interventional cardiology," Catheterization and Cardiovascular Interventions, vol. 70, no. 2, pp. 244-249, 2007.

[86] A. Den Boer, P. J. De Feijter, P. W. Serruys, and J. R. T. C. Roelandt, "Real-time quantification and display of skin radiation during coronary angiography and intervention," Circulation, vol. 104, no. 15, pp. 1779-1784, 2001.

[87] E. Hwang, E. Gaxiola, R. E. Vlietstra et al., "Real-time measurement of skin radiation during cardiac catheterisation," Catheterization and Cardiovascular Diagnosis, vol. 43, pp. 367-370, 1998.

[88] M. Kato, K. Chida, T. Sato et al., "The necessity of follow-up for radiation skin injuries in patients after percutaneous coronary 
interventions: radiation skin injuries will often be overlooked clinically," Acta Radiologica, vol. 53, pp. 1040-1044, 2012.

[89] Radiation Protection of Patients (RPOP), "SAFRAD—Safety in radiological procedures," 2013, https://rpop.iaea.org/safrad.

[90] O. Holmberg, J. Malone, M. Rehani, D. McLean, and R. Czarwinski, "Current issues and actions in radiation protection of patients," European Journal of Radiology, vol. 76, no. 1, pp. 1519, 2010.

[91] M. M. Rehani and D. P. Frush, "Patient exposure tracking: the IAEA smart card project," Radiation Protection Dosimetry, vol. 147, no. 1-2, pp. 314-316, 2011.

[92] R. Padovani and E. Quai, "Patient dosimetry approaches in interventional cardiology and literature dose data review," Radiation Protection Dosimetry, vol. 117, no. 1-3, pp. 217-221, 2006.

[93] K. Faulkner, P. Ortiz-Lopez, and E. Vano, "Patient dosimetry in diagnostic and interventional radiology: a practical approach using trigger levels," Radiation Protection Dosimetry, vol. 117, no. 1-3, pp. 166-168, 2006.

[94] K. A. Fetterly, V. Mathew, R. Lennon et al., "Radiation dose reduction in the invasive cardiovascular laboratory. Implementing a culture and philosophy of radiation safety," Cardiovascular Interventions, vol. 5, pp. 866-873, 2012.

[95] J.-L. Georges, B. Livarek, G. Gibault-Genty et al., "Reduction of radiation delivered to patients undergoing invasive coronary procedures. Effect of a programme for dose reduction based on radiation-protection training," Archives of Cardiovascular Diseases, vol. 102, no. 12, pp. 821-827, 2009.

[96] C. Ubeda, E. Vano, L. Gonzalez et al., "Scatter and staff dose levels in paediatric interventional cardiology: a multicentre study," Radiation Protection Dosimetry, vol. 140, no. 1, pp. 67-74, 2010.

[97] P. L. Khong, H. Ringertz, V. Donoghue et al., "ICRP 121: radiological protection in paediatric diagnostic and interventional radiology," Annals of ICRP, vol. 42, pp. 1-63, 2013.

[98] M. C. Limacher, P. S. Douglas, G. Germano et al., "Radiation safety in the practice of cardiology," Journal of the American College of Cardiology, vol. 31, no. 4, pp. 892-913, 1998.

[99] C. J. Martin, "A review of radiology staff doses and dose monitoring requirements," Radiation Protection Dosimetry, vol. 136, no. 3, pp. 140-157, 2009.

[100] C. P. Shortt, H. Al-Hashimi, L. Malone, and M. J. Lee, "Staff radiation doses to the lower extremities in interventional radiology," CardioVascular and Interventional Radiology, vol. 30, no. 6, pp. 1206-1209, 2007.

[101] D. L. Miller, E. Vañó, G. Bartal et al., "Occupational radiation protection in interventional radiology: a joint guideline of the cardiovascular and interventional radiology society of Europe and the society of interventional radiology," CardioVascular and Interventional Radiology, vol. 33, no. 2, pp. 230-239, 2010.

[102] S. Balter, F. A. Heupler Jr., J. E. Goss et al., "Guidelines for personnel radiation monitoring in the cardiac catheterization laboratory," Catheterization and Cardiovascular Diagnosis, vol. 30, no. 4, pp. 277-279, 1993.

[103] K.-H. Folkerts, A. Münz, and S. Jung, "Estimation of radiation exposure and radiation risk to staff of cardiac catheterization laboratories," Zeitschrift fur Kardiologie, vol. 86, no. 4, pp. 258263, 1997.

[104] K. A. Fetterly, D. J. Magnuson, G. M. Tannahill, M. D. Hindal, and V. Mathew, "Effective use of radiation shields to minimize operator dose during invasive cardiology procedures," Cardiovascular Interventions, vol. 4, no. 10, pp. 1133-1139, 2011.
[105] A. Duran, S. K. Hian, D. L. Miller et al., "recommendations for occupational radiation protection in interventional cardiology," Catheterization and Cardiovascular Interventions, vol. 82, pp. 29-42, 2013.

[106] M. Maeder, H. P. Brunner-La Rocca, T. Wolber et al., "Impact of a lead glass screen on scatter radiation to eyes and hands in interventional cardiologists," Catheterization and Cardiovascular Interventions, vol. 67, no. 1, pp. 18-23, 2006.

[107] R. H. Thornton, L. T. Dauer, J. P. Altamirano, K. J. Alvarado, J. St. Germain, and S. B. Solomon, "Comparing strategies for operator eye protection in the interventional radiology suite," Journal of Vascular and Interventional Radiology, vol. 21, no. 11, pp. 17031707, 2010.

[108] European Commission Directorate-General for the Environment, "Radioprotection 116: Guidelines on education and training in radiation protection for medical exposures," 2000, http:// ec.europa.eu/energy/nuclear/radiation_protection/doc/publication/116.pdf.

[109] E. Vano, M. Rosenstein, J. Liniecki, M. Rehani, C. J. Martin, and R. J. Vetter, "ICRP publication 113: education and training in radiological protection for diagnostic and interventional procedures," Annals of ICRP, vol. 39, 2009.

[110] E. Vaño, L. Gonzalez, J. M. Fernandez, F. Alfonso, and C. Macaya, "Occupational radiation doses in interventionals cardiology: a 15-year follow-up," British Journal of Radiology, vol. 79, no. 941, pp. 383-388, 2006.

[111] V. A. McCormick, C. C. Schultz, V. Hollingsworth-Schuler, J. M. Campbell, W. W. O'Neill, and R. Ramos, "Reducing radiation dose in the cardiac catheterization laboratory by design alterations and staff education," American Journal of Cardiology, vol. 90, no. 8, pp. 903-905, 2002.

[112] MEDRAPET Project ENER/D4/212-2010, "Study on the implementation of the medical exposure directive's requirements on radiation protection training of medical professionals in the European Union," 2012, http://www.medrapet.eu/.

[113] International Atomic Energy Agency (IAEA). Radiation protection of patients (RPOP), https://rpop.iaea.org/RPOP/RPoP/ Content/AdditionalResources/Training/.

[114] E. Vano, "Radiation exposure to cardiologists: how it could be reduced," Heart, vol. 89, no. 10, pp. 1123-1124, 2003.

[115] H. Geijer and J. Persliden, "Radiation exposure and patient experience during percutaneous coronary intervention using radial and femoral artery access," European Radiology, vol. 14, no. 9, pp. 1674-1680, 2004.

[116] M. Sandborg, S.-G. Fransson, and H. Peterson, "Evaluation of patient-absorbed doses during coronary angiography and intervention by femoral and radial artery access," European Radiology, vol. 14, no. 4, pp. 653-658, 2004.

[117] D. Hart, M. C. Hillier, and B. F. Wall, "Doses to patients from medical X-ray examinations in the UK-2000 review," Chilton, NJ, USA, NRPB-W14, 2000.

[118] L. K. Wagner, B. R. Archer, and A. M. Cohen, "Management of patient skin dose in fluoroscopically guided interventional procedures," Journal of Vascular and Interventional Radiology, vol. 11, no. 1, pp. 25-33, 2000.

[119] International Electrotechnical Commission, "Medical electrical equipment-Part 2-43 Ed2.0: particular requirements for the basic safety and essential performance of X-ray equipment for interventional procedures," Report 60601, International Electrotechnical Commission, Geneva, Switzerland, 2010. 

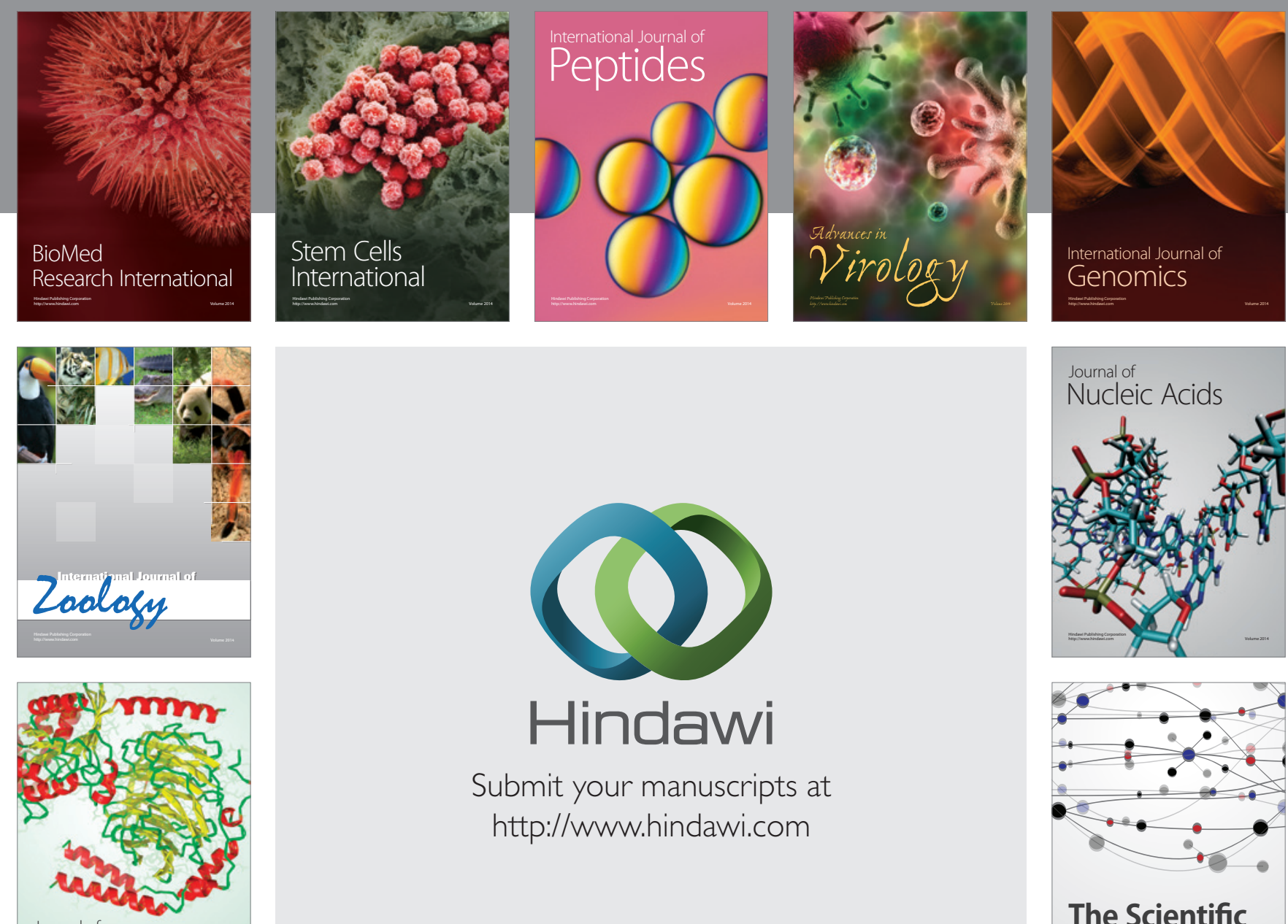

Submit your manuscripts at

http://www.hindawi.com

Journal of
Signal Transduction
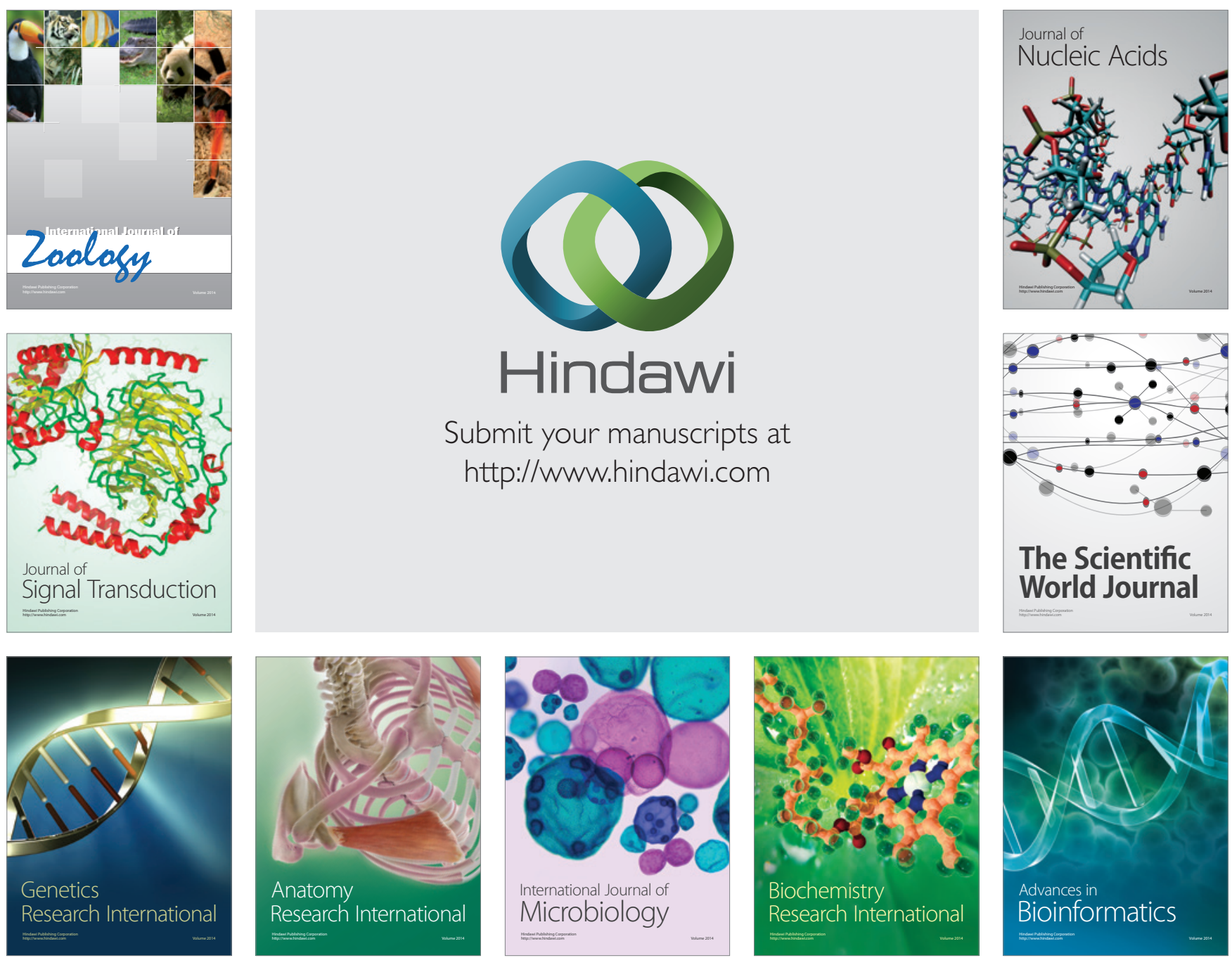

The Scientific World Journal
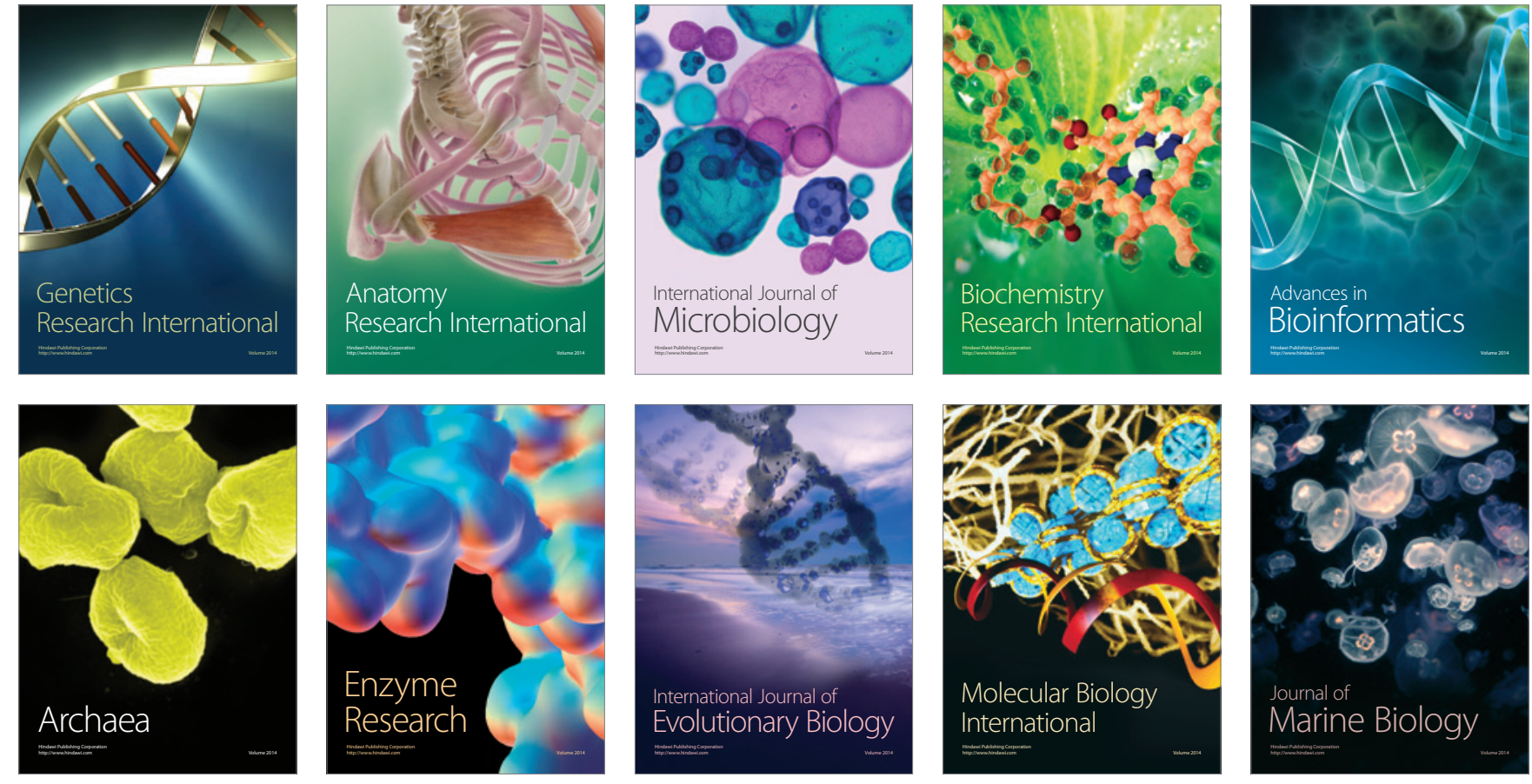